\title{
Optimization of dispersive liquid-liquid microextraction procedure for detecting chlorpyrifos in human urine samples
}

\author{
Maryam Ramin ${ }^{1}$, Monireh Khadem², Fariborz Omidi ${ }^{3}$, Mehran Pourhosein $^{2}$, Farideh Golbabaei², \\ Seyed Jamaleddin Shahtaheri*2,4
}

Received: 11 Mar 2018

Published: 20 Jul 2019

\section{Abstract}

Background: Selecting an effective sample preparation method to measure target pesticides in biological matrices is a serious challenge for researchers. This study aimed to optimize the dispersive liquid-liquid microextraction (DLLME) technique to obtain a simple, valid, and fast method with high efficiency to detect chlorpyrifos in urine samples.

Methods: DLLME, coupled with high performance liquid chromatography equipped with ultra violet detector, was used to extract chlorpyrifos pesticide in human urine samples. Different affecting parameters on the efficiency of the method were optimized using one factor at a time method.

Results: The limit of detection and enrichment factor of the method was 0.5 and $230 \mu \mathrm{g} \mathrm{L}-1$, respectively. Linear calibration curve with 1-500 $\mu \mathrm{g} \mathrm{L-1}$ concentration range was used. The relative standard deviation (RSD) for 6 replicate experiments at the concentration of $200 \mu \mathrm{g} \mathrm{L}-1$ was less than $5 \%$. The relative recoveries of spiked urine samples were $96.3 \%, 102.3 \%$, and $98.7 \%$ at 3 different concentration levels of 50, 200, and $1000 \mu \mathrm{g}$ L-1, respectively.

Conclusion: Compared to other extraction techniques, the optimized DLLME resulted in some advantages such as shorter extraction time, high extraction efficiency, and good enrichment factor for the extraction of chlorpyrifos from human urine samples.

Keywords: Chlorpyrifos, Dispersive liquid-liquid microextraction, Urine, HPLC

Conflicts of Interest: None declared

Funding: Tehran University of Medical Sciences

\section{*This work has been published under CC BY-NC-SA 1.0 license.}

Copyright $\odot$ Iran University of Medical Sciences

Cite this article as: Ramin M, Khadem M, Omidi F, Pourhosein M, Golbabaei F, Shahtaheri SJ. Optimization of dispersive liquid-liquid microextraction procedure for detecting chlorpyrifos in human urine samples. Med J Islam Repub Iran. 2019 (20 Jul);33:71. https://doi.org/10.47176/mjiri.33.71

\section{Introduction}

Organophosphate pesticide poisoning is commonly found among toxin-producing workers in factories, toxin spraying workers, and consumers of contaminated foods and equipment $(1,2)$.

Most farmers who use pesticides daily are at risk due to the lack of proper knowledge about poisoning of these

Corresponding author: Dr Seyed Jamaled din Shahtaheri, shahtaheri@tums.ac.ir

1. Occupational and Environmental Center, Health deputy, Ministry of Health and Medical Education, Tehran, Iran

2. Department of Occupational Health Engineering, School of Public Health, Tehran University of Medical Sciences, Tehran, Iran

3. Research Center for Environmental Determinants of Health (RCEDH), Kermanshah University of Medical Sciences, Kermanshah, Iran

4. Institute for Environmental Research, Tehran University of Medical Sciences, Tehran, Iran chemicals and correct ways of working with them (3). Therefore, it is necessary to develop appropriate techniques to extract these hazardous compounds from biological samples to evaluate the exposure of workers.

Conventional sample preparation techniques, such as liquid-liquid extraction (LLE), solid-liquid extraction

$\uparrow$ What is "already known" in this topic:

Most sample preparation techniques have been used in combination with gas chromatography as a high selective or sensitive detection system, which are expensive and cannot be used widely for the analysis of organophosphorus pesticides in developing countries.

\section{$\rightarrow$ What this article adds:}

The proposed method in this study, without any special detector, is a sensitive, simple, rapid, and repeatable sample preparation method and can be used for the extraction and preconcentration of chlorpyrifos residues from aqueous samples. 
(SLE), and soxhlet extraction, are widely accepted and used for routine applications. Furthermore, new studies are being conducted in this field with the aim of developing faster and stronger preparation and extraction techniques (4-7). Solvent microextraction (SME) is a method for preparing samples by extraction and condensation of liquid, gas, and solid samples using $100 \mu \mathrm{L}$ solvent or less. The SME is used to extract, purify, and concentrate the volatile, nonvolatile, polar, nonpolar, ionic, and metallic analytes from environmental, biological, and agricultural samples. There are several types of SMEs that are generally divided into 2 major categories: (1) exposed to solvent, and (2) solvent with membrane (7-9)

Dispersive liquid-liquid microextraction (DLLME), hollow-fiber microextraction (HFME), single-drop microextraction (SDME), and liquid-liquid microextraction (LLME) were the main and most widely used methods of the SME procedure in recent years. Unlike other sample preparation methods, SME is still used for scientific research (10-13).

DLLME, which belongs to the category of exposed solvents, is widely used as a sample preparation method with high extraction recovery (14-17). Extracting solvent, insoluble in water, is dissolved in dispersive solvent, then, it is rapidly injected into the liquid sample by a proper syringe. The rapid injection of a mixture of organic solvents into the water causes the insoluble solvent to be extracted rapidly in small microdrops from the target analyte. The enriched organic phase is separated from the liquid sample by centrifuging and it is directly injected to the analyzing instrument (18-21).

Chlorpyrifos (trade name of Dursban EC40.8\%), as a subgroup of organic phosphorus insecticides and phosphorothioic acid is used to control a wide range of agricultural and domestic pests (17). This pesticide has nonsystemic, gastro-intestinal, and respiratory effects (22-25). The durability of this compound in the soil is 2 to 4 months, and its toxicity is very high for humans and animals. The ability of this poison to inhibit cholinesterase enzyme in the nervous system has attracted much attention to control and reduce workers' exposures (26-29).

The present study aimed to optimize DLLME as a fast, effective, low-cost, and simple extraction method to determine the trace amounts of chlorpyrifos in human urine samples. In this study, a useful sample preparation method, with high extraction of very few pesticide residue in the matrix, was developed.

\section{Methods}

\section{Reagents and solutions}

Chlorpyrifos with purity of more than $98 \%$ were provided by Dr. Ehrenstorfer Company (Germany). Organic solvents, including carbon tetrachloride, carbon disulfide, chloroform, methanol, acetonitrile, and acetone, were purchased from Merck (Darmstadt, Germany). Analyticalreagent grade sodium chloride, hydrochloric acid, and sodium hydroxide were also obtained from Merck. Deionized water was purchased from Behan Company (Tehran, Iran). A stock solution of chlorpyrifos (1000 ppm) was prepared by dissolving an appropriate amount of the pesti- cide in acetonitrile. Working standard solutions were prepared daily by diluting the stock solution with deionized water.

\section{Instrumentation}

HPLC (HPLC pump k-1001, UV detector k-2600; Knauer, Japan), equipped with a UV detector, was used to determine and separate chlorpyrifos. The separation was performed on Agilent Eclipse Plus C18 column ( $\mathrm{L}=250$ $\mathrm{mm}, \mathrm{ID}=4.6 \mathrm{~mm}$; Reprosil-PUR C-18 AQ $10 \mu \mathrm{m}$ ) using methanol-water solution $(60: 40, \mathrm{v} / \mathrm{v})$ as mobile phase. The pump flowrate and column temperature were set at $1.5 \mathrm{~mL} / \mathrm{min}$ and $25^{\circ} \mathrm{C}$, respectively. The chromatographic response for the analyte and matrix interference was acceptable under the detection wavelength of $203 \mathrm{~nm}$. A Hettich zentrifugen Rotofix 32 (Baoding, China) was used for centrifugation. The samples were ultrasonically irradiated in water bath at $150 \mathrm{~W}$ and $40 \mathrm{kHz}$ using an ultrasonic equipment (SonoSwiss SW $6 \mathrm{H}$ ). All glassware used in the experiments were washed with acetone and deionized water and dried in an oven at $50^{\circ} \mathrm{C}$ temperature.

\section{Dispersive liquid-liquid microextraction procedure}

First, $10 \mathrm{~mL}$ spiked urine sample with defined concentration of analyte $(1 \mathrm{ppm})$ was poured into a $15 \mathrm{~mL}$ centrifugal tube; then, $1.5 \mathrm{~mL}$ of methanol containing $150 \mu \mathrm{L}$ carbon tetrachloride was quickly injected to the centrifugal tube. The cloudy solution was centrifuged for $5 \mathrm{~min}$ at $4000 \mathrm{rpm}$ and the extractant was settled to the bottom of centrifugal tube. The phase containing chlorpyrifos was separated by a syringe and poured into another test tube, and its solvent evaporated under the gentle flow of $\mathrm{N}_{2}$. Finally, the remaining settled phase was dissolved in methanol and $20 \mu \mathrm{L}$ of it was withdrawn using a $100 \mu \mathrm{L}$ microsyringe and injected into the HPLC for quantification (Fig. 1).

Eight factors that could potentially affect the chlorpyrifos extraction were examined to find their optimum levels. These factors included the extraction solvent, disperser solvent, volume of the extraction solvent, volume of the disperser solvent, centrifugation time and speed, salt addition, and sample $\mathrm{pH}$. In each step, 7 factors were constant and 1 varied in different levels to determine the optimum quantity. Figure 2 shows chromatograms of aqueous sample of 1 ppm chlorpyrifos before (A) and after (B) of ap-

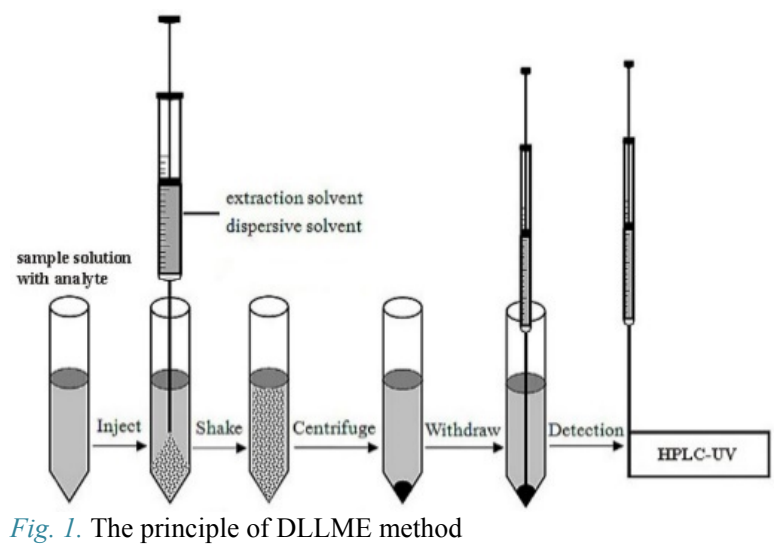


(A)

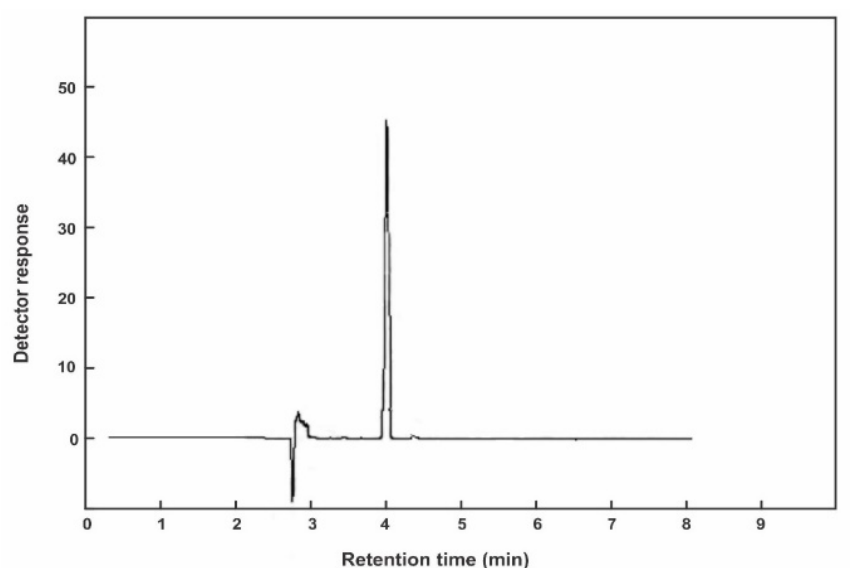

Fig. 2. Chromatograms of aqueous sample of chlorpyrifos before (A) and after (B) preconcentration
(B)

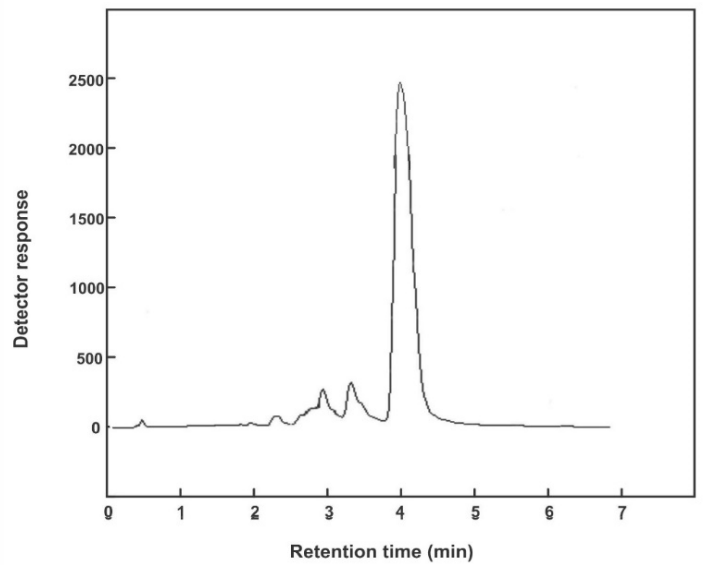

Retention time ( $\mathrm{min})$ plying DLLME procedure.

\section{Urine sample preparation}

Urine samples were collected from exposed workers and stored in a freezer at $-18^{\circ} \mathrm{C}$. Urine samples $(5.0 \mathrm{~mL})$ were placed in centrifuge tubes and diluted with $50 \mathrm{~mL}$ double-distilled water. PH was adjusted at 6 by adding sodium hydroxide solutions to the samples. Next, the prepared specimen was analyzed according to the proposed preparation method.

\section{Enrichment factor and extraction recovery}

To develop DLLME method for preconcentration of chlorpyrifos, some parameters controlling the extraction efficiency were investigated using sample solutions with the analyte concentration of $1 \mathrm{ppm}$. To evaluate the extraction efficiency, enrichment factor (EF) and extraction recovery (ER) of the analyte were calculated by the following equations:

$$
\mathrm{EF}=\mathrm{C}_{\mathrm{sed}} / \mathrm{C}_{0}
$$

Where, $\mathrm{C}_{\text {sed }}$ and $\mathrm{C}_{0}$ are the concentrations of the analyte in the settled phase and in the aqueous samples before extraction, respectively.

$\mathrm{C}_{\text {sed }}$ was obtained from the calibration curve of direct injection of standard solutions.

$\mathrm{ER}=\mathrm{C}_{\text {sed }} \mathrm{V}_{\text {sed }} / \mathrm{C}_{0} \mathrm{~V}_{\mathrm{aq}} \times 100 \%=\mathrm{EF} \times\left(\mathrm{V}_{\text {sed }} / \mathrm{V}_{\mathrm{aq}}\right) \times 100 \%$

Where, $\mathrm{V}_{\text {sed }}$ and $\mathrm{V}_{\mathrm{aq}}$ are the volumes of the settled phase and the aqueous sample, respectively. The average of 3 replicate extractions was reported for all experiments.

\section{Results}

\section{Effect of extraction solvent}

An extraction solvent was selected based on capability to extract the analyte as well as its appropriate chromatographic behavior. Three solvents, including carbon tetrachloride $\left(\mathrm{CCl}_{4}\right)$, carbon disulfide $\left(\mathrm{CS}_{2}\right)$, and chloroform
$\left(\mathrm{CHCL}_{3}\right)$, were examined as extraction solvents in DLLME. The important properties of selected solvents, such as density and solubility, could affect the extraction efficiency of the target analyte. For this purpose, $10 \mathrm{~mL}$ aqueous solutions of chlorpyrifos (1 ppm) were used to optimize the extraction solvent. According to the obtained results, no distinct cloudy solution was formed using $\mathrm{CS}_{2}$ and $\mathrm{CHCL}_{3}$ as extraction solvents, indicating that they could not effectively disperse among aqueous sample solution because of low extraction capability. Contrasted with $\mathrm{CS}_{2}, \mathrm{CHCL}_{3}, \mathrm{CCl}_{4}$ resulted in the highest extraction efficiency for chlorpyrifos. Hence, $\mathrm{CCl}_{4}$ was selected as the optimum extraction solvent for subsequent experiments.

\section{Effect of disperser solvent}

The type of disperser solvent is very important for obtaining preconcentration of the analyte. The chosen solvents must be appropriately miscible in both extraction solvent and sample solution, so that they can form a distinct cloudy solution. Therefore, 4 possible disperser solvents, including methanol, ethanol, acetonitrile, and acetone, were examined. Methanol showed the highest extraction recovery for the analyte compared to other mentioned solvents. Taking into account the data, methanol was selected for later experiments.

\section{Effect of volume of extraction solvent}

The effect of extraction solvent volume on the enrichment factor and extraction recovery of the analyte was evaluated by using $2 \mathrm{~mL}$ methanol containing different volumes of $\mathrm{CCl}_{4}(50,100,150$, and $200 \mu \mathrm{L})$. With the increase of the $\mathrm{CCl}_{4}$ volume, the extraction recoveries of chlorpyrifos increased. It was also found that, the volume of the sediment phase at the bottom of the test tube increased by elevating the volume of $\mathrm{CCl}_{4}$ from 50 to $150 \mu \mathrm{L}$. According to the results, more volume of $\mathrm{CCl}_{4}$ led to the highest extraction recovery and after that it was constant. Therefore, the volume of $150 \mu \mathrm{L}$ was selected as the optimal volume of $\mathrm{CCl}_{4}$. 
Effect of volume of disperser solvent

The other parameter that could affect the extraction recovery and enrichment factor was the volume of disperser solvent. Different methanol volumes $(0.5,1,1.5,2,2.5$ $\mathrm{mL}$ ) containing $150 \mu \mathrm{L} \mathrm{CCl}_{4}$ were used to find the optimal volume. The extraction recoveries of the analyte increased at first and then decreased by raising the volume of methanol. At low volumes of methanol, the cloudy solution was not formed completely, leading to the low extraction recovery of the analyte. When the volume of methanol increased to $1.5 \mathrm{~mL}$, the highest extraction recovery and enrichment factor was obtained due to decreasing the solubility of $\mathrm{CCl}_{4}$ in aqueous solution. However, the extraction recovery decreased when the volume of methanol was more than $1.5 \mathrm{~mL}$. This decrease can be explained with the increase in the ratio of the dispersive solvent to the extraction solvent and prevention in settling the extraction solvent.

According to the results, $1.5 \mathrm{~mL}$ methanol was chosen to obtain high enrichment factor and extraction recovery.

\section{Effect of centrifugation time and speed}

To separate the extractant phase, an important factor in DLLME is centrifugation process. This step destroys the cloudy solution and helps the extraction solvent to settle at the bottom of the tube. The effects of centrifugation time and rate on the extraction efficiency were examined and optimized in the ranges of 5-20 min and 2500-4000 rpm, respectively. According to the results, the time of 5 minutes and the speed of $4000 \mathrm{rpm}$ were selected as the optimum levels of centrifugation for next experiments.

\section{Effect of salt addition}

To prove the effect of ionic strength on the extraction efficiency, different concentrations of sodium chloride $(0$, 2,4 , and $6 \% \mathrm{w} / \mathrm{v}$ ) were investigated. Salting out can decrease the solubility of the analyte in the aqueous phase and increase extraction into the organic phase. Based on the obtained results, increasing the $\mathrm{NaCl}$ concentration led to the lower extraction efficiency, as the increase of the aqueous phase viscosity decreased the diffusion coefficient of the analyte. Therefore, next experiments were performed with no salt addition.

\section{Effect of sample $\mathrm{pH}$}

Sample $\mathrm{pH}$ is also another important factor affecting the extraction efficiency. By adding the appropriate amount of hydrochloric acid or sodium hydroxide solutions to water samples, the stability of chlorpyrifos under the $\mathrm{pH}$ range of 2-10 was tested. It was indicated that the higher extraction recovery of the analyte was obtained at $\mathrm{pH} 6$. Therefore, doubled-distilled water was used without $\mathrm{pH}$ adjustment in the study.

\section{Analysis}

Analytical features of the method: The analytical characteristics of the method, including linear range (LR), limit of detection (LOD or MDL), limit of quantification (LOQ), correlation coefficient $\left(\mathrm{r}^{2}\right)$, relative standard deviation ( $\mathrm{RSD} \%)$, enrichment factor $(\mathrm{EF})$, and efficiency recovery (ER), were determined under the optimized conditions to evaluate the performance of the method. The obtained results were summarized in Table 1. linearity was over a broad concentration range for the pesticide, with correlation coefficient $\left(\mathrm{r}^{2}\right)$ of 0.993 . The MDL and LOQ, calculated based on signal to noise ratio $(\mathrm{S} / \mathrm{N})$ of 3 and 10 , were 0.5 and 5 , respectively. The RSD values were less than $5 \%$ for interday and intraday precisions, indicating an acceptable repeatability for the developed method. The EF and ER for the pesticide were 230 and $95.7 \%$, respectively. Satisfactory repeatability, high EF and ER, and low MDL and LOQ are the main advantages of the proposed method.

Urine sample analysis: The proposed method was applied to the preconcentration and determination of chlorpyrifos in the spiked urine samples. To validate the accuracy of the DLLME procedure, samples were spiked with the target analyte at 3 different concentration levels of 50, 200, and $1000 \mu \mathrm{g} \mathrm{L}^{-1}$ and analyzed in triplicate using the recommended method. The analyte recoveries are shown in Table 2. According to obtained data, the relative recoveries ranged from $96.3 \%-102.3 \%$. The relative recoveries of the analyte indicated no significant differences in concentration levels of 50,200 , and $1000 \mu \mathrm{g} \mathrm{L}^{-1}$, confirming the validity of the proposed method. The obtained RSDs for the real samples were fairly low at different concentrations. These results indicate that the real sample matrix has no significant effect on the proposed method for preconcentration of chlorpyrifos from urine sample.

\section{Discussion}

The comparison of LR, RSD, MDL, LOQ and EF obtained for the presented method with those of other reported methods for analysis of the target analyte in different samples is summarized in Table 3. The RSD of the proposed method is comparable or better than those reported for the other methods. This study found lower MDL and LOQ compared to most reported methods. The MDL was

Table 1. Quantitative features of the proposed method for chlorpyrifos
LR

\begin{tabular}{|c|c|c|c|c|c|c|c|}
\hline $\begin{array}{c}\mathrm{LR} \\
\left(\mu \mathrm{g} \mathrm{L}^{-1}\right)\end{array}$ & $r^{2}$ & $\begin{array}{c}\operatorname{MDL}(\mu \mathrm{g} \\
\left.\mathrm{L}^{-1}\right)\end{array}$ & $\begin{array}{c}\mathrm{LOQ} \\
\left(\mu \mathrm{g} \mathrm{L}^{-1}\right)\end{array}$ & $200 \mu \mathrm{g} \mathrm{I}$ & $(n=6)$ & $\mathrm{EF}$ & ER (\%) \\
\hline $5-500$ & 0.9931 & 0.5 & 5 & $\begin{array}{c}\text { Intra-day } \\
1.9\end{array}$ & $\begin{array}{c}\text { Inter-day } \\
4.69\end{array}$ & 230 & 95.7 \\
\hline
\end{tabular}

Table 2. Relative recovery (RR) and RSD values of chlorpyrifos in urine sample

\begin{tabular}{ccc} 
& \\
\hline Spiked levels $\left(\mu \mathrm{g} \mathrm{L}^{-1}\right)$ & $\mathrm{RR}(\%)$ & $\mathrm{RSD}(\%)(\mathrm{n}=3)$ \\
\hline 50 & $96.3 \pm 2.1^{\mathrm{a}}$ & 2.2 \\
200 & $102.3 \pm 4.2$ & 4.07 \\
1000 & $98.7 \pm 3.1$ & 3.1 \\
\hline
\end{tabular}

${ }^{a}$ Mean of three determinations \pm standard deviation 
M. Ramin, et al.

Table 3. Comparison of the presented method with other methods used in the analysis of the target analyte

\begin{tabular}{|c|c|c|c|c|c|c|c|c|c|}
\hline Pesticide & Sample & MDL & LOQ & $\begin{array}{l}\text { RSD } \\
(\%)\end{array}$ & LR & $\begin{array}{l}\text { Extraction } \\
\text { Factor }\end{array}$ & $\begin{array}{l}\text { Extraction } \\
\text { time (min) }\end{array}$ & Method & Reference \\
\hline Diazinon & Water & $1.4 \mu \mathrm{g} \mathrm{L}^{-1}$ & - & 9.4 & $5-500 \mu \mathrm{g} \mathrm{L}^{-1}$ & - & 20 & PN-SDME- & $(30)$ \\
\hline Chlorpyrifos & sample & $1.6 \mu \mathrm{g} \mathrm{L}^{-1}$ & - & 8.3 & $5-500 \mu \mathrm{g} \mathrm{L}^{-1}$ & & & GC-MS & \\
\hline Malathion & & $1.6 \mu \mathrm{g} \mathrm{L}^{-1}$ & - & 7 & $5-500 \mu \mathrm{g} \mathrm{L}^{-1}$ & & & & \\
\hline Diazinon & Aqueous & $0.65 \mu \mathrm{g} \mathrm{L}^{-1}$ & $2.2 \mu \mathrm{g} \mathrm{L}^{-1}$ & 6 & $3-40000 \mu \mathrm{g} \mathrm{L}^{-1}$ & - & 15 & MWA- & (31) \\
\hline Chlorpyrifos & sample & $0.74 \mu \mathrm{g} \mathrm{L}^{-1}$ & $2.5 \mu \mathrm{g} \mathrm{L}^{-1}$ & 4 & $3-40000 \mu \mathrm{g} \mathrm{L}^{-1}$ & & & DLLME- & \\
\hline Malathion & & $1.3 \mu \mathrm{g} \mathrm{L}^{-1}$ & $4.5 \mu \mathrm{g} \mathrm{L}^{-1}$ & 4 & $3-40000 \mu \mathrm{g} \mathrm{L}^{-1}$ & & & GC-FID & \\
\hline Diazinon & Summer & $2 \mu \mathrm{g} \mathrm{kg}^{-1}$ & $6.3 \mu \mathrm{g} \mathrm{kg}^{-1}$ & 5.5 & $5-500 \mu \mathrm{g} \mathrm{kg}^{-1}$ & 50 & 35 & UASE- & (32) \\
\hline Chlorpyrifos & crops & $4 \mu \mathrm{g} \mathrm{kg}^{-1}$ & $11.8 \mu \mathrm{g} \mathrm{kg}^{-1}$ & 9.1 & $10-800 \mu \mathrm{g} \mathrm{kg}^{-1}$ & & & $\begin{array}{c}\text { DLLME- } \\
\text { SFO- } \\
\text { HPLC- UV }\end{array}$ & \\
\hline Diazinon & Fruit juice & $0.5 \mu \mathrm{g} \mathrm{kg}^{-1}$ & - & 1.51 & $4-200 \mu \mathrm{g} \mathrm{kg}^{-1}$ & 50 & 40 & CPE-GC- & (33) \\
\hline Chlorpyrifos & & $1 \mu \mathrm{g} \mathrm{kg}^{-1}$ & - & 2.27 & $4-200 \mu \mathrm{g} \mathrm{kg}^{-1}$ & & & FPD & \\
\hline Malathion & & $1 \mu \mathrm{g} \mathrm{kg}^{-1}$ & - & 1.88 & $4-200 \mu \mathrm{g} \mathrm{kg}^{-1}$ & & & & \\
\hline Chlorpyrifos & Urine & $0.5 \mu \mathrm{g} \mathrm{L}^{-1}$ & $5 \mu \mathrm{g} \mathrm{L}^{-1}$ & 4.07 & $5-500 \mu \mathrm{g} \mathrm{L}^{-1}$ & 230 & 4 & $\begin{array}{l}\text { DLLME- } \\
\text { HPLC-UV }\end{array}$ & This work \\
\hline
\end{tabular}

lower or comparable than most of the mentioned techniques even those employing mass spectrometry (MS) detectors. Most of the mentioned techniques have used a high selective or sensitive detection system, such as flame photometric detection (FPD), flame ionization detector (FID), or MS; and all of these detectors in combination with gas chromatography (GC) are expensive and cannot be widely used to analyze organophosphorus pesticides in developing countries. The proposed method in this study does not need any special detector, but it still is sensitive, simple, rapid, and repeatable and can be used for the extraction and preconcentration of chlorpyrifos residues from aqueous samples.

In a study done by Wang et al in 2011 (30), pneumatic nebulization single-drop microextraction method was used to determine organophosphorus pesticides by gas chromatography-mass spectrometry. The results showed that MDL and RSD were $1.6 \mu \mathrm{g} \mathrm{L}^{-1}$ and 8.3, respectively. However, in the present study, MDL and RSD were 0.5 $\mu \mathrm{g} \mathrm{L}^{-1}$ and 4.07, which were much lower. Also, in studies conducted by Farajzadeh et al in 2016 (31), ionic liquids that are lighter than water were used as extraction solvents and achieved favorable and very similar results in the present study.

In this study, DLLME procedure was successfully developed from aqueous samples for the extraction of chlorpyrifos. The most important effective factors for the extraction of the analyte were investigated and optimized. The proposed procedure had some advantages in comparison with other extraction techniques such as shorter extraction time, better reproducibility, and higher enrichment factor. Also, good precision, suitable recoveries, broad dynamic linear range, and low limit of detection were attained using the DLLME method.

\section{Conclusion}

In the present work, DLLME procedure was successfully optimized for the extraction of chlorpyrifos from human urine samples. Different effecting factors in extraction of the analyte were investigated and developed. The proposed optimized procedure in comparison with the other extraction techniques had more advantages such as shorter extraction time, better reproducibility, and higher enrichment factor. The worthy analytical figures, such as suitable recoveries, good precision and enrichment factor, wide dynamic linear range, and low limit of detection, were attained by DLLME method. In conclusion, according to obtained results, the proposed method can be used as a simple procedure to provide high preconcentration efficiency to determine chlorpyrifos in complex matrices.

\section{Acknowledgements}

This work has been extracted from Ph.D. thesis of the first author, Maryam Ramin (research code: of 9223491001). The authors would like to thank Tehran University of Medical Sciences for funding and supporting this project. This study received funding from Tehran University of Medical Sciences.

\section{Conflict of Interests}

The authors declare that they have no competing interests.

\section{References}

1. Pawliszyn J. Sampling and Sample Preparation in Field and Laboratory. Fundamentals and New Directions in Sample Preparation (Comprehensive Analytical Chemistry); 2002.

2. Wang H, Li G, Zhang Y, Chen H, Zhao Q, Song W, et al. Determination of triazine herbicides in cereals using dynamic microwaveassisted extraction with solidification of floating organic drop followed by high-performance liquid chromatography. J Chromatogr A. 2012;1233(1):36-43.

3. Koohpaei AR, Shahtaheri SJ, Ganjali MR, Rahimi Forushani A, Golbabaei F. Application of multivariate analysis to the screening of molecularly imprinted polymers (MIPs) for ametryn. Talanta. 2008;75(4): 978-986.

4. Amelin V, Lavrukhin D, Tret yakov A. Dispersive liquid-liquid microextraction for the determination of herbicides of urea derivatives family in natural waters by HPLC. J Anal Chem. 2013;68(9):822-830.

5. Xiao Y, Zhang H. Homogeneous ionic liquid microextraction of the active constituents from fruits of Schisandra chinensis and Schisandra sphenanthera. Anal Chim Acta. 2012;712(1):78-84.

6. Andruch V, Acebal CC, Skrlikova J, Sklenarova H, Solich P, Balogh IS et al. Automated on-line dispersive liquid-liquid microextraction based on a sequential injection system. Microchem J. 2012;100(1):7782.

7. Ramos L. Critical overview of selected contemporary sample preparation techniques. J Chromatogr A. 2012;1221(1):84-98.

8. Kokosa JM. Advances in solvent-microextraction techniques. Trends Anal Chem. 2013;43(1):2-13.

9. Gjelstad A, Jensen H, Rasmussen KE, Petersen-Bjergaard S. Kinetic aspects of hollow fiber liquid-phase microextraction and electromembrane extraction. Anal Chim Acta. 2012;742(1):6-10. 
10. Breadmore MC. Ionic liquid-based liquid phase microextraction with direct injection for capillary electrophoresis. J Chromatogr A. 2011; 1218(10):1347-1352.

11. Anastassiades M, Lehotay SJ, Stajnbaher D, Schenck FJ. Fast and easy multi-residue method employing acetonitrile extraction/ partitioning and dispersive solid-phase extraction for the determination of pesticide residues in produce. J AOAC Int. 2003;86(2):412-431.

12. Fritz JS, Macka M. Solid-phase trapping of solutes for further chromatographic or electrophoretic analysis. J Chromatogr A. 2000; 902(1):137-166.

13. Behbahani M, Hassanlou PG, Amini M, Omidi F, Esrafili A, Farzadkia $\mathrm{M}$ et al. Application of solvent-assisted dispersive solid- phase extraction as a new, fast, simple and reliable preconcentration and trace detection of lead and cadmium ions in fruit and water samples. Food Chem. 2015; 187(1):82-88.

14. Rezaee M, Assadi Y, Milani Hosseini MR, Aghaee E, Ahmadia F, Berijani S. Determination of organic compounds in water using dispersive liquid-liquid microextraction. J Chromatogr A. 2006;1116(2):1-9.

15. Jamali MR, Firouzjah A, Rahnama R. Solvent-assisted dispersive solid- phase extraction. Talanta. 2013;116(1):454-459.

16. Zhang YF, Lee HK. Low-density solvent-based vortex-assisted surfactant-enhanced-emulsification liquid-liquid microextraction combined with gas chromatography-mass spectrometry for the fast determination of phthalate esters in bottled water. J Chromatogr A. 2013; 1274(1):28-35.

17. Barata C, Solayan A, Porte C. Role of B-esterases in assessing toxicity of organophosphorus (chlorpyrifos, malathion) and carbamate (carbofuran) pesticides to Daphnia magna. Toxicol. 2004;66(2):125-139.

18. Omidi F, Behbahani M, Bojdi MK, Shahtaheri SJ. Solid phase extraction and trace monitoring of cadmium ions in environmental water and food samples based on modified magnetic nanoporous silica. J Magn Magn Mater. 2015;395(1):213-220.

19. Cruz-Vera M, Lucena R, Cardenas S, Valcarcel M. Sample treatments based on dispersive (micro) extraction. Anal Method. 2011; 1(8):1719-1728.

20. Pourhossein M, Shahtaheri SJ, Maleck Khani H, Rahimi Foroushani A, Seyed Someah M, Divani R. Optimization of dispersive liquid liquid microextraction method for determination of trace salivary melatonin using high performance liquid chromatography. Iran Occup Heal J. 2017;14(4):85-94.

21. Ho YM, Tsoi YK, Leung KSY. Highly sensitive and selective organophosphate screening in twelve commodities of fruits, vegetables and herbal medicines by dispersive liquid-liquid microextraction. Anal Chim Acta. 2013;775(2):58-66.

22. Yan H, Du J, Zhang X, Yang G, Row KH, Lv Y. Ultrasound-assisted dispersive liquid-liquid microextraction coupled with capillary gas chromatography for simultaneous analysis of nine pyrethroids in domestic wastewaters. J Sep Sci. 2010;33(12):1829-1835.

23. Cinelli G, Avino P, Notardonato I, Russo MV. Ultrasound-vortexassisted dispersive liquid-liquid microextraction coupled with gas chromatography with a nitrogen-phosphorus detector for simultaneous and rapid determination of organophosphorus pesticides and triazines in wine. Anal Methods. 2014;3:782-790.

24. Sanagi MMM, Miskam SU, Ibrahim MA, Wan WA. Determination of organophosphorus pesticides by dispersive liquid-liquid micro extraction coupled with gas chromatography-electron capture detection. Malays J Anal Sci. 2011;15(2):232-239.

25. Sousa R, Homem V, Moreira JL, Madeira LM, Alves A. Optimisation and application of dispersive liquid-liquid microextraction for simultaneous determination of carbamates and organophosphorus pesticides in waters. Anal Methods. 2013;11:2736-2745.

26. Wu C, Liu H, Liu W, Wu Q, Wang C, Wang Z. Determination of organophosphorus pesticides in environmental water samples by dispersive liquid-liquid microextraction with solidification of floating organic droplet followed by high-performance liquid chromatography. Anal Bioanal Chem. 2010;397(6):2543-2549.

27. Mukdasai S, Thomas C, Srijaranai S. Two-step microextraction combined with high performance liquid chromatographic analysis of pyrethroids in water and vegetable samples. Talanta. 2014; 120(1):289-296.

28. Boonchiangma S, Ngeontae W, Srijaranai S. Determination of six pyrethroid insecticides in fruit juice samples using dispersive liquidliquid microextraction combined with high performance liquid chromatography. Talanta. 2012;88(1):209-215.

29. Shan H. Simultaneous determination of six neonicotinoid residues in soil using DLLME-HPLC and UV. Spectrosc Spect Anal. 2013; 33(9):2553-2557.

30. Pirsaheb M, Fattahi N, Shamsipur M. Determination of organophosphorous pesticides in summer crops using ultrasound-assisted solvent extraction followed by dispersive liquid-liquid microextraction based on the solidification of floating organic drop. Food Control. 2013; 34(2):378-385.

31. Zhao WJ, Sun XK, Deng XN, Huang L, Yang MM, Zhou ZM. Cloud point extraction coupled with ultrasonic-assisted back-extraction for the determination of organophosphorous pesticides in concentrated fruit juice by gas chromatography with flam photometric detection. Food Chem. 2011;127(2):683-688.

32. Wang Y, Wang Z, Zhang H, Shi Y, Ren R, Zhang H et al. Application of pneumatic nebulization single-drop microextraction for the determination of Organophosphorous pesticides by gas chromatographymass spectrometry. J Sep Sci. 2011;34(15):1880-1885.

33. Farajzadeh MA, Asghari A, Feriduni B. An efficient, rapid and microwave-accelerated dispersive liquid-liquid microextraction method for extraction and pre-concentration of some organophosphorus pesticide residues from aqueous samples. J Food Compos Anal. 2016; 48(1):73-80. 